\title{
Calculated Neutron-Source Spectra from Selected Irradiated PWR Fuel Assemblies
}

\author{
P. Mi. Rinard* \\ G. E. Bosler \\ J. R. Phillips
}

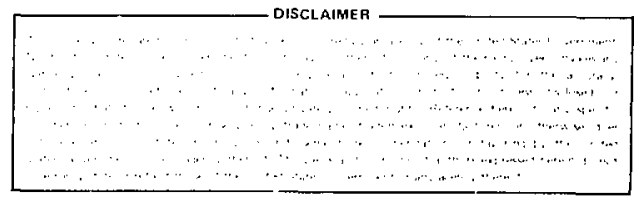

* Short-Term Visiting Staff Member. Department of Physics, Emporia State University. Emporia, KS 66801.

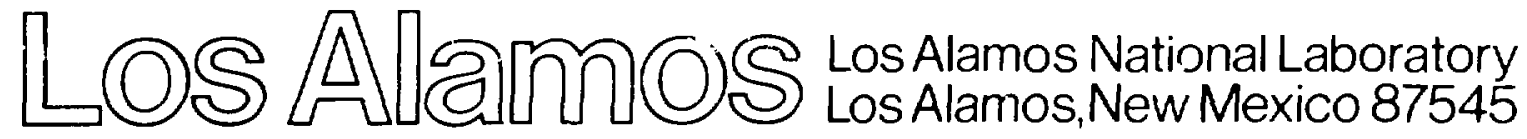




\title{
CALCULATED NEUTRON SOURCE SPECTRA FROM SELECTED IRRADIATED PWR FUEL ASSEMBLIES
}

by

P. M. Rinard, G. E. Bosler, and J. R. Phillips

\begin{abstract}
The energy spectra of neutrons emitted from a pressurized-water-reactor fuel assembly have been calculated for a variety of exposures and cooling times. They are presented in graphical form. Some effects of initial enrichment are also included.

Neutrons from spontaneous fissions were given either a Maxwellian temperature of 1.2 or $1.5 \mathrm{MeV}$, depending on whether they were due to plutonium and uranium nuclides or curium nuclides. A single $(a, n)$ spectrum was deemed sufficient to represent the neutrons from all the alpha-emitting nuclides.

The proportions of the nuclides undergoing spontaneous fission and those emitting alpha particles were determined from calculated atom densities. The particular pressurizedwater-reactor fuel assembly assumed for this purpose was of the type used in the H. B. Robinson Unit-2 power plant (740 MWe).
\end{abstract}

\section{INTRODUCTION}

Neutrons emitted from an irradiated fuel assembly can assist in determining the exposure and fissile content of the assembly. ${ }^{1}$ The interpretation of a detector's response must start with knowledge of the emission rate and energy spectrum of the neutrons being produced within the assembly. These quantities can depend on the initial enrichment, the exposure history, and the

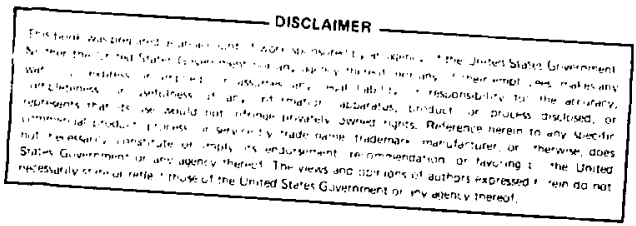


cooling time. The spectra presented in this report illustrate the effects of these three parameters. The additional concern of the transport of the neutrons to a detector will not be addressed here.

knowledge of the spectra can be of value in the evaluations of measurements taken during safeguards inspections, in the design of storage containers for irradiated fuels, and in the general problem of shielding for the fuels.

While there can be many types of fuels with different geometries and irradiation histories, a common type of pressurized-water-reactor (PWR) fuel assembly can be represented by that of the H. B. Robinson Unit-2 reactor. This assemily forms the basis for this study.

I. CALCULATIONAL METHID

A. Atom Densities

The primary sources of neutrons in irradiated assemblies are spontaneous fissions and $(\alpha, n)$ reactions with oxygen. The $(r, n)$ reactions contribute an unimportant fraction of the total neutrons for fuel assemblies with more than a few weeks cooling time. ${ }^{1}$ Uranium and transuranic nuclides are the princiFal emitters of neutrons, so their atom densities must be known.

A study ${ }^{2}$ of a fuel assembly used in the H. B. Robinson Unit-2 740-Mwe Westinghouse reactor provided the desirad atom densities. A combination of the EPRI-CELL ${ }^{3}$ and EPRI-CINDER ${ }^{4}, 5$ codes was applied to the 15 by 15 fuel pin array of this PWR. Most of the spectra shown in this report assume an initial ${ }^{235} \mathrm{U}$ enrichment of $2.561 \%$ (typical for the $H . B$. Robinson PWR), although comparisons with enrichments of 3.5 and $4.5 \%$ are given. The power level was constant in time, with a linear power density of $200 \mathrm{~W} / \mathrm{cm}$.

\section{B. Spontaneous Fission Spectra}

The Maxwellian curve with its "temperature" parameter T was assumed to represent a fission spectrum adequately. When normalized to an area of one, it can be multiplied by the specific emission rate $n_{\text {sf }}$ to give the number of neutrons released per unit volume per unit time per energy interval:

$$
\frac{d_{n}}{n}=\frac{\sqrt{E} e^{-E / T}}{0.886227 T^{3 / 2}} d E,
$$


where $d_{n}$ is the number of neutrons with energies in the range of $E$ to $E+d E$ and $n$ is the total number of neutrons.

The value of $T$ that best fits the data for $240_{\mathrm{Pu}}$ is $1.19 \mathrm{MeV}{ }^{6}$ such measurements for other nuclides are not available, so assistance was sought from calculated spectra. 7 It appears that the plutonium and uranium nuclides of interest $\mathrm{c}$ an be assigned a spectral temperaturt: of $1.2 \mathrm{MeV}$, while $1.5 \mathrm{MeV}$ is more suitable for the ${ }^{242} \mathrm{Cm}$ and ${ }^{244} \mathrm{Cm}$ ruclides. These are the temperatures used in the present calculation, and the resulting normalized spectra are shown in Fig. 1. Fortunately, the fission spectrum is nct strongly sensitive to small changes in $T$, so that these two assumed spectra should be adequate in providing a useful estimate of the desired spectral shapes.

C. $(a, n)$ Spectra

The alpha particles from ${ }^{238} \mathrm{Pu},{ }^{239} \mathrm{Pu}$, and $240 \mathrm{Pu}$ are generally the most numerous ones in the fuel, and neutron spectra due to their reactions with ${ }^{17} 0$ and ${ }^{18} 0$ have been studied. ${ }^{8-11}$ The alpha particle energies for these nuclides are 5.5, 5.2, and 5.15 MeV, respectively. Their measured and caiculated spectra are so similar that one curve can represent them all. Because ${ }^{238} P_{u}$ is the largest $(\alpha, n)$ contributor to the overall spectra, its spectrum is used for all the plutonium nuclides.

Another important alpha emitter when the exposure is above $3000 \mathrm{MWd} / \mathrm{tU}$ is ${ }^{241} \mathrm{Am}$. Data on the neutron spectrum it generates with oxygen are unavailable, but because its alpha particle has an energy of $5.5 \mathrm{MeV}$ its spectrum is assumed to be the same as that of ${ }^{238} \mathrm{Pu}$.

The only other actinides of importance are ${ }^{242} \mathrm{~cm}$ and ${ }^{244} \mathrm{Cm}$. Ayain, data on their neutron spectra are unavailable. The alpha particle energy of ${ }^{244} \mathrm{~cm}$ is $5.8 \mathrm{MeV}$, so the ${ }^{238} \mathrm{Pu}$ spectrum is assumed to be valid for ${ }^{244} \mathrm{Cm}$. The case of ${ }^{242} \mathrm{Cm}$ is not so simple because its alpha particle has a high energy of $6.12 \mathrm{MeV}$. In addition to extending the theoretical maximum neutron energy, additional excited states of the daughter nucleus are accessible and may affect the spectrum. Not only are there no data for this case, there seems to be no direct information on the effect of such a high-energy alpha particle on oxygen. Beryllium, however, has been the target of alpha particles from four nuclides, 12,13 including ${ }^{242} \mathrm{Cm}$. The fcur alpha particle energies were between 4.8-6.12 MeV; there are only minor differences in the neutron spectra. The 
spectrum due to ${ }^{242} \mathrm{Cm}$ does not appear to differ from the others in any important way in the case of this target.

With the oxygen target, the higher excited states in the daughter neon may not have any impact on the spectrum because calculated spectra have been quite accurate wh.en only the first two excited states are included. The higher the energy of the final daughter state, the less frequently it is involved in the reaction.

It is thus assumed that the ${ }^{238} \mathrm{Pu}$ spectrum may be used for the ${ }^{242} \mathrm{Cm}$ neutrons as well as for all other alpha emitters in this study. This approximation is further justific:d by calculating the maximum fraction of all neutrons that result from ${ }^{242} \mathrm{Cm}$. The largest value found with $2.561 \%$ enrichment occurs at an exposurf of $11000 \mathrm{MWd} / \mathrm{tU}$ and zero cooling time; yet even this maximum fraction is only $12 \%$ of the total number of neutrons present. The fraction is much less after shorter exposures because ${ }^{242} \mathrm{Cm}$ is produced in smaller amounts. The fraction rapidly decreases with cooling time (regardless of the exposure) because of the short half-life of ${ }^{242} \mathrm{~cm}$. With exposures above 11000 $\mathrm{MWd} / \mathrm{tU}$ (and a cooling time of zero), the production of ${ }^{244} \mathrm{Cm}$ increases and the fraction of neutrons from ${ }^{242} \mathrm{Cm}$ decreases.

The ${ }^{238} \mathrm{Pu}$ spectrum used for all the neutrons, regardless of the source or the alpha particle, follows the available data except at high and low energies where only the calculated spectra suggest the shape. The normalized shape has three segments and is multiplied by the total rate $\mathrm{N}_{\alpha, n}$ :

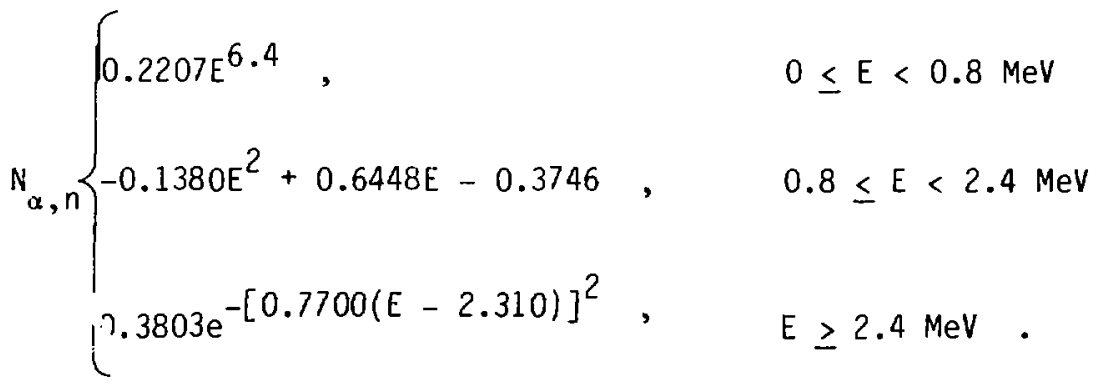

The normalized curve is shown in Fig. 1 and can be easily compared to the spontaneous fission spectra. 


\section{Total Neutron Spectra}

To obtain the total neutron spectra (in units of number of neutrons per cubic centimeter per second), sums of Eqs. (1) and (2) were taken. The total neutron proruction rate from the spontaneous fissions of all plutonium and uranium nuclides gave a value for $n_{s f}$ in Eq. (1) when $T=1.2 \mathrm{MeV}$, and the total rate from the curium nuclides gave another $n_{s f}$ for $T=1.5 \mathrm{MeV}$. For the third contribution to the total neutron spectra, all the neutron production rates from individual nuclides were added to give the $N_{\alpha, n}$ in Eq. (2). The three curves in Fig. 1 were thus given weighting factors according to the production rate of neutrons from the three processes involved. A sum of these three weighted curves gives the total spectrum.

\section{RESULTS}

\section{A. Effect of Exposure}

Figures 2-4 show the spectra as functions of exposure and cooling time for the base case with $2.561 \%$ enrichment. Spontaneous fissions always account for more than one-half of the neutrons, easily dominating the $(a, n)$ neutrons in irradiated fuels with exposures above $10000 \mathrm{MWd} / \mathrm{tU}$.

Near zero exposure, the spontaneous fission neutrons are almost all due to ${ }^{238} \mathrm{U}$, while ${ }^{242} \mathrm{Cm}$ and ${ }^{244} \mathrm{Cm}$ provide the bulk of the neutrons at the higher exposures. With exposures between 750-10000 MWd/tU, the $\{\alpha, n\}$ neutrons from ${ }^{238} \mathrm{Pu}$ and ${ }^{241} \mathrm{Am}$ are nearly equal in number to all of those from spontaneous fission. Above the highest exposure shown, the spectra are almost entirely due to the spontaneous fissioning of ${ }^{244} \mathrm{Cm}$, with some contribution from ${ }^{242} \mathrm{Cm}$ if the cooling time is less than 24 months.

When the spontaneous fission fraction is less than 0.8 , the spectra show a tendency to form two maxima. The lower energy "maximum" is nearly all from the plutonium spontaneous fissions, while the higher energy "maximum" is from the sum of these spontaneous fissions and the $(\alpha, n)$ reactions. At higher exposures, enough curium spontaneous fission neutrons are produced to almost completely dominate the spectra. 


\section{B. Effect of Cooling Time}

Figure 5 shows how cooling time alters the spectra after different exposures. For exposures below $2500 \mathrm{MWd} / \mathrm{tU}$, the cooling time makes little difference because long-lived plutonium nuclides are the most prominent sources. At higher exposures, the cooling time has a larger effect. This is especially true for the first year or two, because significant anounts of ${ }^{242} \mathrm{Cm}$ are produced, with a half-life of 163 days.

The effects of both exposure and cooling time are summarized in Fig. 6, where the fraction of the neutrons due to spontaneous fission is plotted as a function of the exposure and cooling time.

At zero exposure, the ${ }^{238} U$ spontaneous fissions provide nearly all the neutrons. Around $1000 \mathrm{MWd} / \mathrm{tU}$, the maximum fraction of neutrons from $(a, n)$ reactions occurs (due to ${ }^{238} \mathrm{Pu}$ and ${ }^{239} \mathrm{Pu}$ ), but it is still just under one-half of the total. For less than $11000 \mathrm{MWd} / \mathrm{tU}$, the spontaneous fission fraction decreases monotonically with cooling time; above that exposure, the fraction increases for about 2 or $3 \mathrm{yr}$, then decreases very slowly. This transition takes place when ${ }^{244} \mathrm{Cm}$ begins to be produced in large amounts.

Another representation of this information is the ratio of spontaneous fission neutrons to $(a, n)$ neutrons, as shown in Fig. 7. The change in the effect of cooling time just discussed $c$ an be seen in this figure also. Along a vertical line for some exposures $<11000 \mathrm{MWd} / \mathrm{tU}$, the curves for shorter cooling times lie above those for longer cooling times. Above $11000 \mathrm{MWd} / \mathrm{tU}$, the sequence is reversed.

\section{Effect of Enrichment}

Comparisons of spectra from assemblies with enrichments of 2.561, 3.5, and $4.5 \%$ are given in Fig. 8 . In this study, higher enrichment gives a lower curve because the atom density calculations wer: for a fixed power level, leading to a lower neutron fluence to achieve the same exposure. A lower flluence (higher initial ${ }^{235} U$ enrichment) would result in less of the transuraric isotopes being built up for the same exposure.

\section{Isotopic Fractions}

The effects of various nuclides on the number of the neutrons produced as a function of exposure and cooling time are shown in Fig. 9. The fractions of all the neutrons due to the five largest contributors are shown for different 
exposures and cooling times; these five contributions account for more (usually much more) than $90 \%$ of all the neutrons. The changing importance of the nuclides can be seen in the figure. As the exposure increases, the dominating nuclide shifts from uranium, to plutonium, and then to curium. Cooling time has a secondary effect compared to exposure. Once again, the exposure near $11000 \mathrm{Md} / \mathrm{tU}$ is a transition point. Just below that exposure, the plutonium nuclides are dominant, while just above it the curium nuclides are the major producers. Very near $11000 \mathrm{MWd} / \mathrm{tU}$, the curium and plutonium importances vary with cooling time.

\section{E. Comparison to Another Study}

A similar study has been reported ${ }^{14}$ on Russian light-water reactors (WWER) of 365 and 440 MWe power with fuel enriched to $3 \%$. While the general approach to calculating the spectra was similar to the one given here, there are some differences in the details that lead to minor but noticeable effects.

The spectra fresented in Ref. 14 show a much stronger tendency to form two maxima, but with the higher peak at the lower energy. The more pronounced maxima resulted from use of the normalized spectra for spontaneous fission and $(a, n)$ reactions.

The $(a, n)$ reaction spectrum of Ref. 9 was used in the Russian work; it is a "harder" spectrum than indicated by the data 10,11 and the calculated spectrum of Ref. 8.

The treatment of the spontaneous fission neutrons was not completely explained in REf. 14, but ic seerns that spectral curves from ${ }^{240} \mathrm{Pu}$ and ${ }^{244} \mathrm{Cm}$ were used to generate a single curve to represent all the spontaneous fission emissions. The only spontaneous fission spectrum presented was for ${ }^{244} \mathrm{Cm}$; it had a Maxwellian temperature of $1.35 \mathrm{MeV}$. This value is lower than that used in the present calculations and is more appropriate for induced fissions.

With a harder $(\alpha, n)$ spectrum and a lower Maxwellian temperature, the curves in Fig. 1 would be farther apart and the two maxima in Fig. 2 more distinct. This explains the main difference between these two studies. We believe our choice of spectra in Fig. 1 is more appropriate because the Maxwellian temperatures are especially selected for spontaneous fissions and the $(a, n)$ spectrum is in better accord with the measured values.

One further comparison that can be made is the change of the spontaneous fission fraction with cooling time after a fairly large exposure. The Russian 
calculation for WWER 3\%-enriched fuel after an exposure of $30000 \mathrm{MWd} / \mathrm{tU}$ shows the fraction rapidly increasing for $\sim 2 \mathrm{yr}$, then reaching a maximum after $3 \mathrm{yr}$ and very slowly decreasing. For the $2.561 \%$-enriched fuel used here, and after sn exposure of $33000 \mathrm{MWd} / \mathrm{tU}$, the same rapid increase in the spontaneous fission fraction is found (Fig. 7). Instead of reaching a maximum, however, the fraction continues to increase at a very slow rate.

The choice of normalized spectra affects the average energies so that our values are larger than those used for the Russian study, especially after a high exposure. Their sirgle spontaneous fission spectrum is somewhat of an average of our two, but when the curium sources are dominant, their spectrum is "softer" than ours. Their "harder" $(\alpha, n)$ spectrum does not make up the difference because the $(\alpha, n)$ reactions produce a minority of the neutrons. For example, immediately after an exposure of $30000 \mathrm{MWd} / \mathrm{tU}$, our average energy is $2.25 \mathrm{MeV}$, while theirs is $2.14 \mathrm{MeV}$. After a cooling time of 36 months, our average energy is practically unchanged, but theirs is reduced to $2.07 \mathrm{MeV}$.

\section{REFERENCES}

1. J. R. Phillips, J. K. Halbig, D. M. Lee, S. E. Beach, T. R. Bement, E. Dermendjiev, C. R. Hatcher, K. Kaieda, and E. G. Medina, "Application of Nondestructive Gamma-Ray and Neutron Techniques for the Safeguarding of Irradiated Fuel Materials," Los Alamos Scientific Laboratory report LA-8212 (ISP0-77) (May 1980).

2. W. B. Wilson, R. J. LaBauve, and T. R. England, "H. B. Robinson-2 Spent Fuel Isotopics; Sensitivity Series 2" (T-2-1128), Los Alamos Scientific Laboratory memo to John Phillips and Gerald Bosler (October 9, 1980).

3. W. R. Cobb and W. J. Ejch, "A New Cell Depletion Code," Trans. Am. Nucl. Soc. 24, $442(1976)$.

4. T. R. England, W. B. Wilson, and M. G. Stamatelatos, "Fission Product Data for Thermal Reactors, Part 2: Users' Manual for EPRI-CINDER Code and Data," Electric Power Research Institute report EPRI NP-356, Part 2 (December 1976); also published as Los Alamos Scientific Laboratory report LA-6746-MS (December 1976).

5. T. R. England, W. B. Wilson, and M. G. Stamatelatos, "Fission Product Data for Thermal Reactors, Part 1: A Data Set for EPRI-CINDER Using ENDF/B-IV," Electric Power Research Institute report EPRI NP-356, Part 1 (December 1976); also published as Los Alamos Scientific Laboratory report LA-6745-MS (December 1976). 
6. P. R. Fields and H. Diamond, "Californium-252, A irimary Standard for Neutrons," Neutron Dosimetry (IAEA, Vienna, 1963), Vol. II, p. 585.

7. D. G. Madland, "Calculation of Prompt Fission Neutron Spectra for Cm-242(sf) and Cm-244(sf)" (T-2-1129), Los Alamos Scientific Laboratory memo to Gerald Bosler (October 10, 1980).

8. D. L. Lessor and R. E. Schenter, "Neutron Spectra from $(\alpha, n)$ Reactions in Plutonium Compounds Calculated from Hauser-Feshback Reaction Theory," WADCO Corp. 189, No. 12519 (June 1971).

9. M. Taherzadeh, "Neutron Yield from the $(\alpha, n)$ Reaction in the Isotope 0xygen-18," Nucl. Sci. and Eng. 44, 190-193 (1971).

10. M. E. Anderson, "Neutron Energy Spectra of 239pu-Be, 238pu-F, and 238pu-180 $(\alpha, n)$ Sources," Mound Laboratory report MLM-1422 (October 31, 1967).

11. A. G. Khabakhpashev, Soviet J. At. Energy, English Trans 1., 7, 591 (1960).

12. S. Notarrigo, F. Porto, A. Rubbino, and S. Sambataro, "Experimental and Calculated Energy Spectra of Am-Be and Pu-Be Neutron Sources," Nucl. Phys. A125, 28-32 (1969).

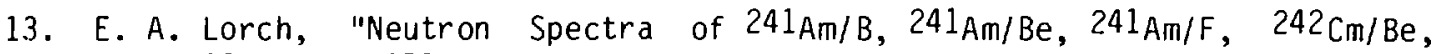
$233 \mathrm{Pu} /{ }^{13} \mathrm{C}$, and ${ }^{252} \mathrm{Cf}$ Isotopic Neutron," Int. J. Appl. Radiat. Isot. 24 , 585-591 (1973).

14. N. S. Shemanskya, "Neutron Energy Spectra of Spent Fuel from WWER Reactors," Sov. J. At. Energy 49, 351 (1980). 


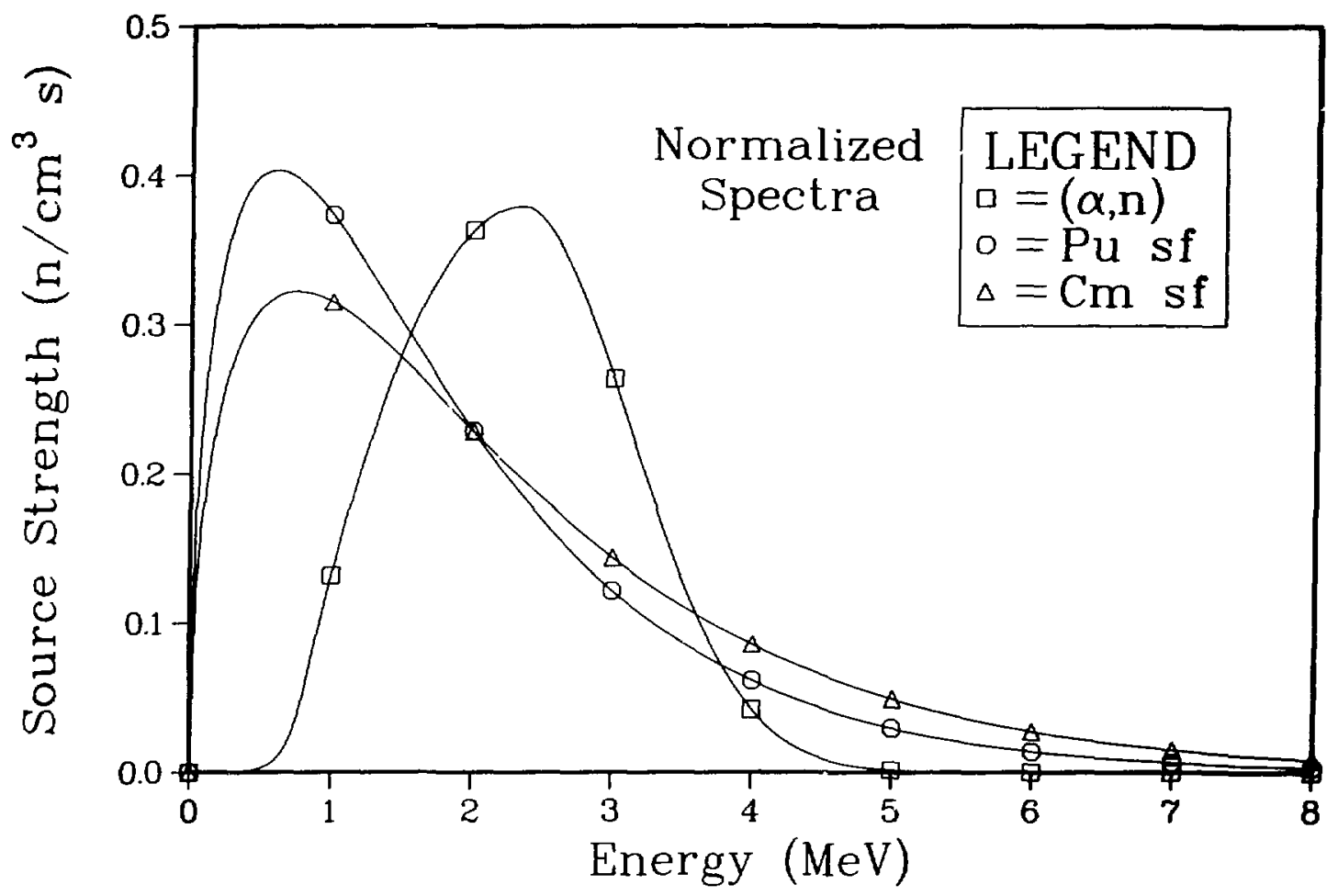

Fig. 1 .

The neutron spectra given in Figs. 2-5 consist of sums of these three curves in proportions determined by calculated atom densities. Ail spectra from $(\alpha, n)$ reactions with oxygen are given the same shape, but two different spontaneous fission spectra are used (one for plutonium and uranium nuclides, another for cur ium nuclides). 

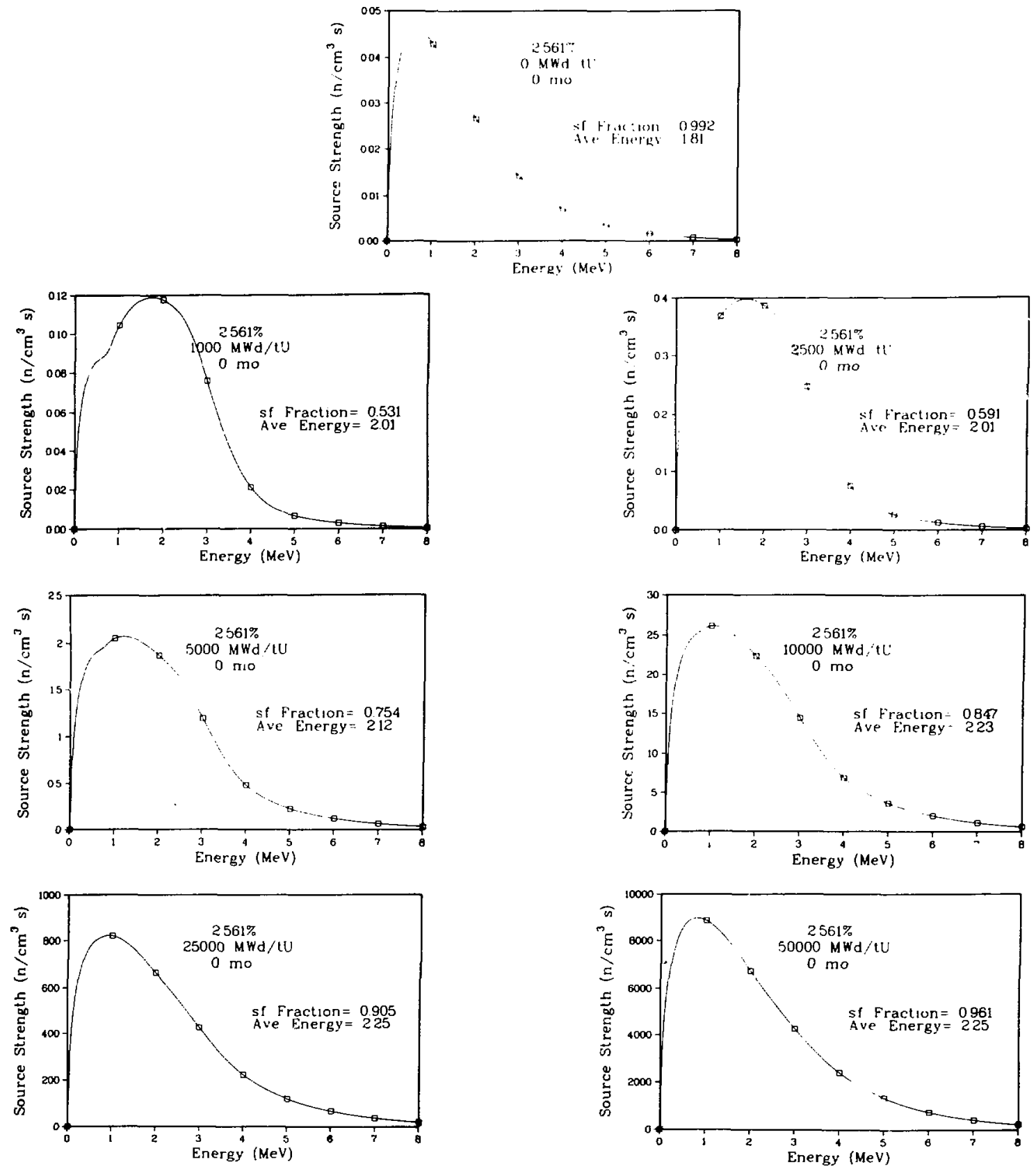

Fig. 2.

The neutron spectrum is affected by the exposure given the $2.561 \%$-enriched fuel. These graphs show the spectrum as the exposure is increased. Each graph has a grouping of numbers giving the initial enrichment, the exposure, and the cooling time. Throughout this figure, the cooling time is zero. Also provided is the fraction of the nextrons originating from spontaneous fission events. 

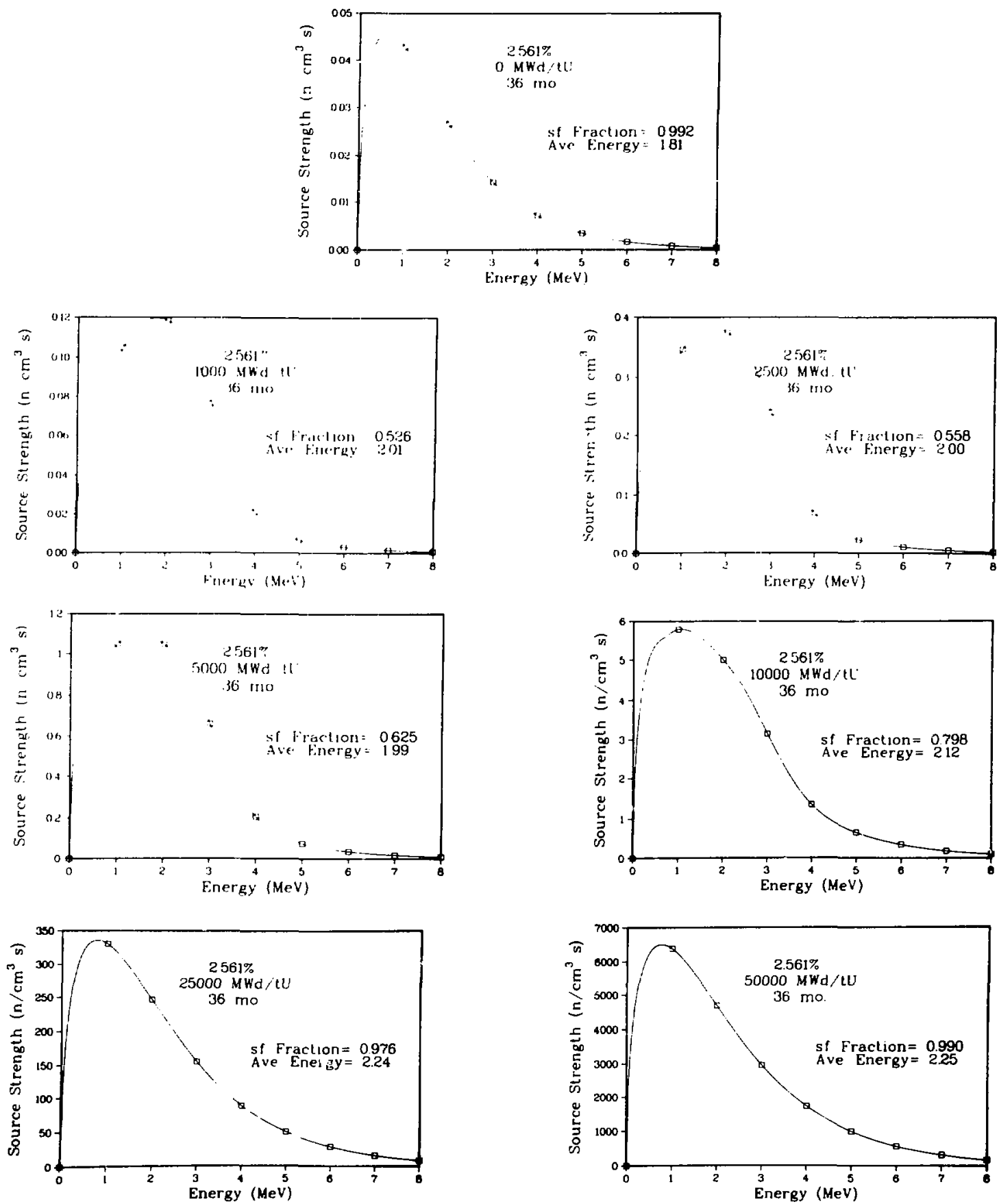

Fig. 3 .

Refer to the caption of Fig. 2, but ncte that the cooling time is now 36 months. 

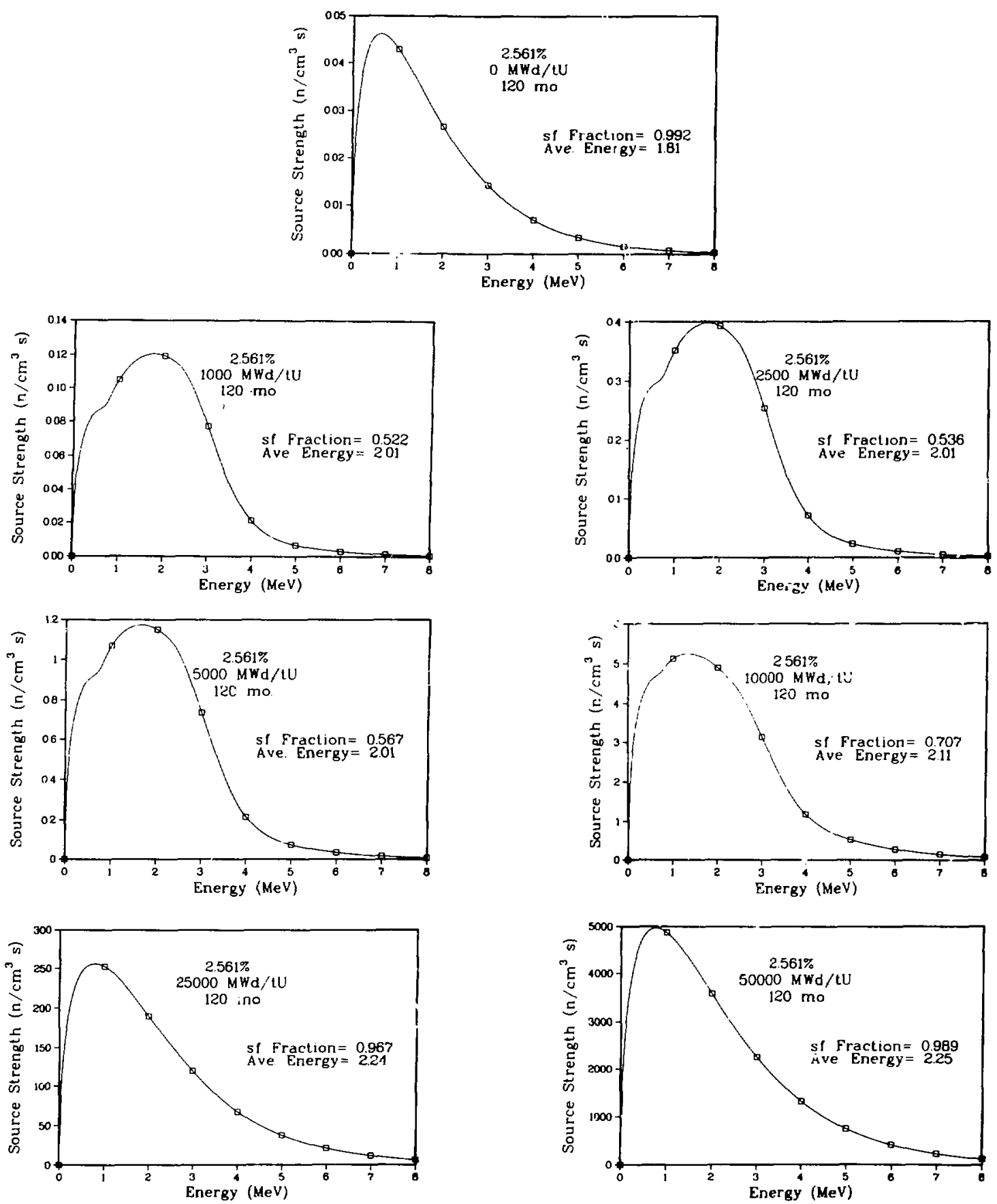

Fig. 4 .

Refer to the caption of Fig. 2, but note that the cooling time is now 120 months. 

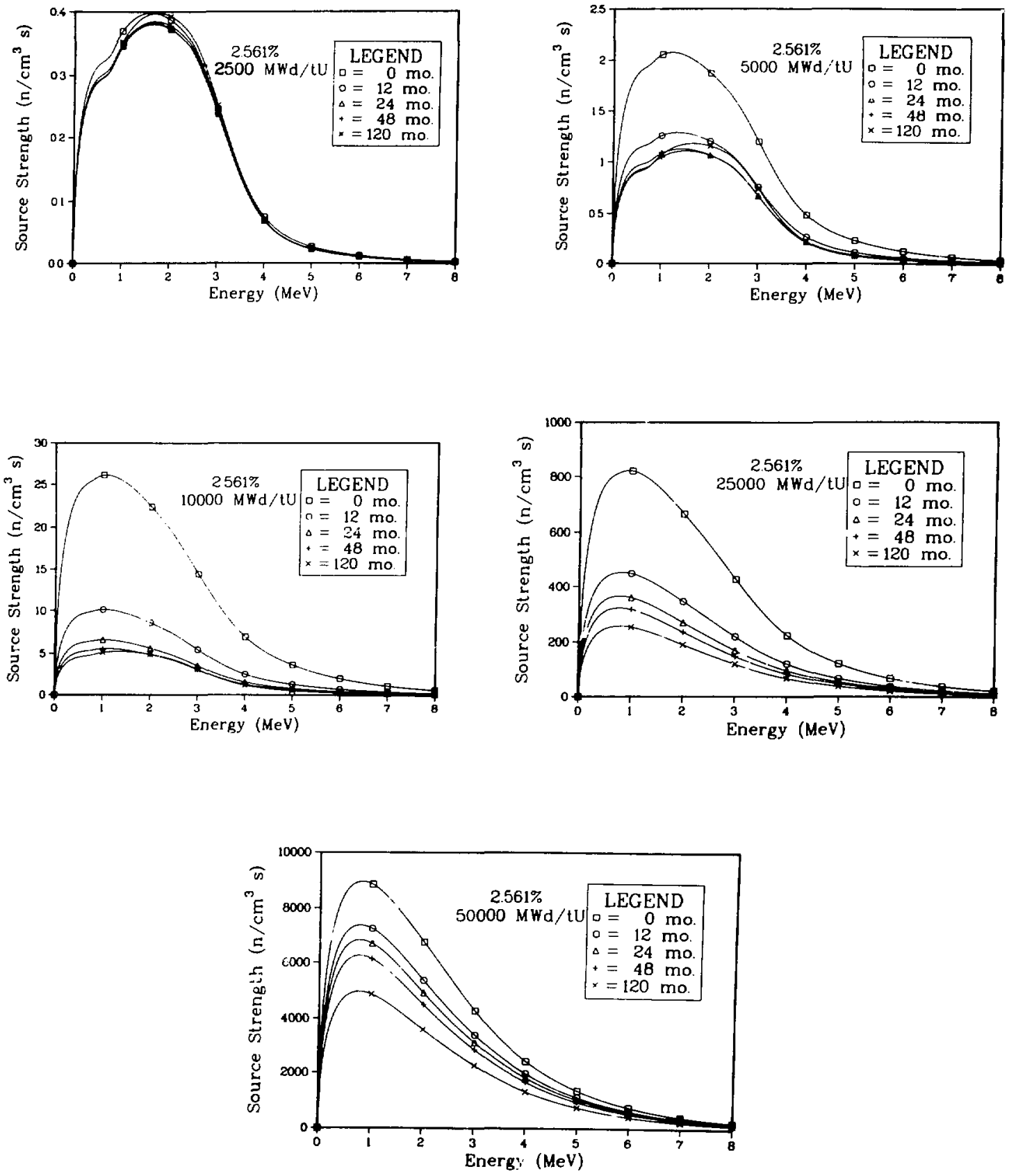

Fig. 5 .

The effect of cooling time on the neutron spectrum can be seen in this figure. For each of five exposures, the spectra after five cooling times are given. 

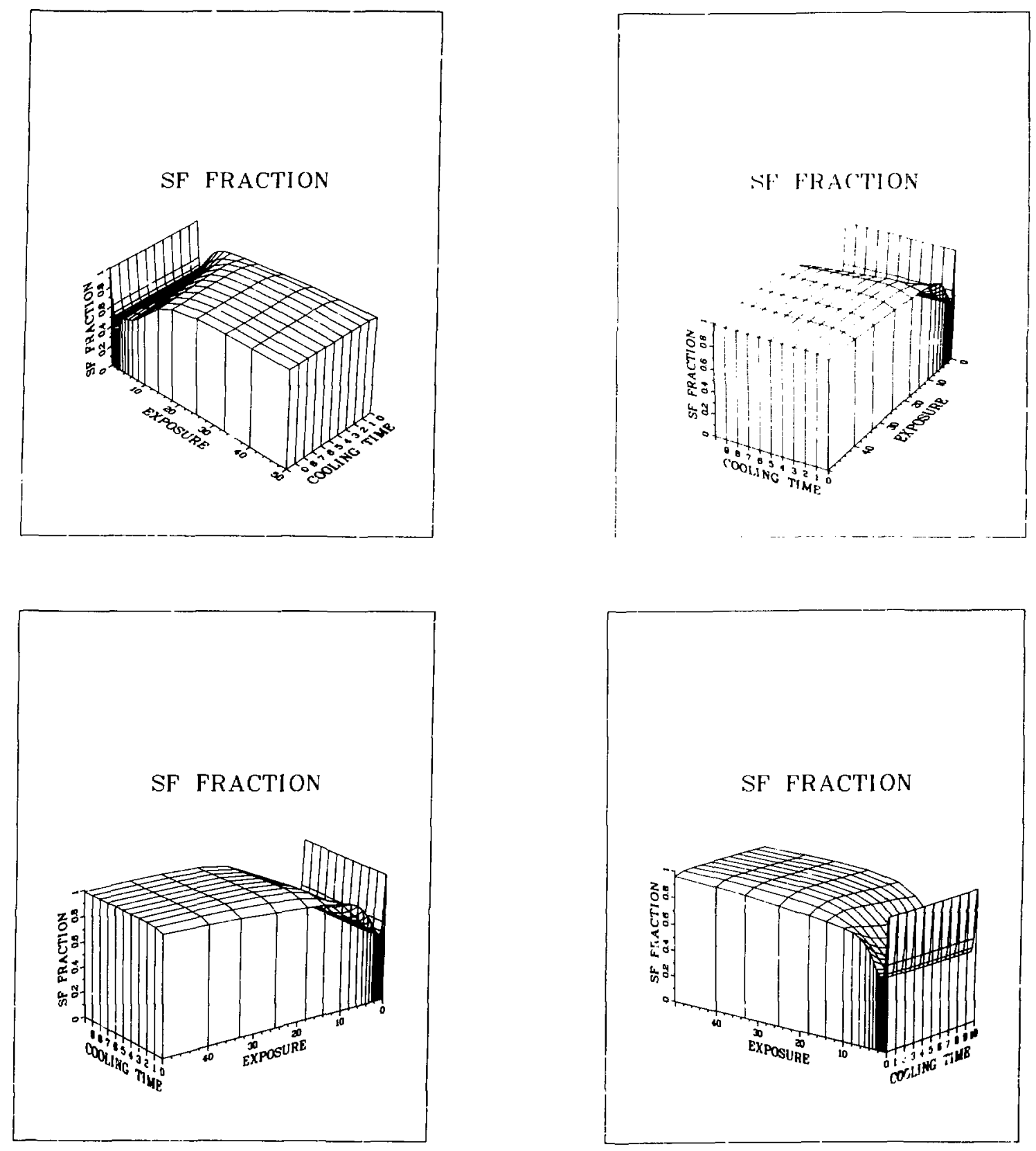

Fig. 6 .

These are four views of the same surface of the fraction of neutrons due to spontaneous fission, as a function of exposure (in GWd/tU) and cooling time (in years). Notice that after an exposure below $\sim 1$ GWd/tU, the fraction decreases with cooling time; above $11 \mathrm{GWd} / \mathrm{tU}$, it increases. 


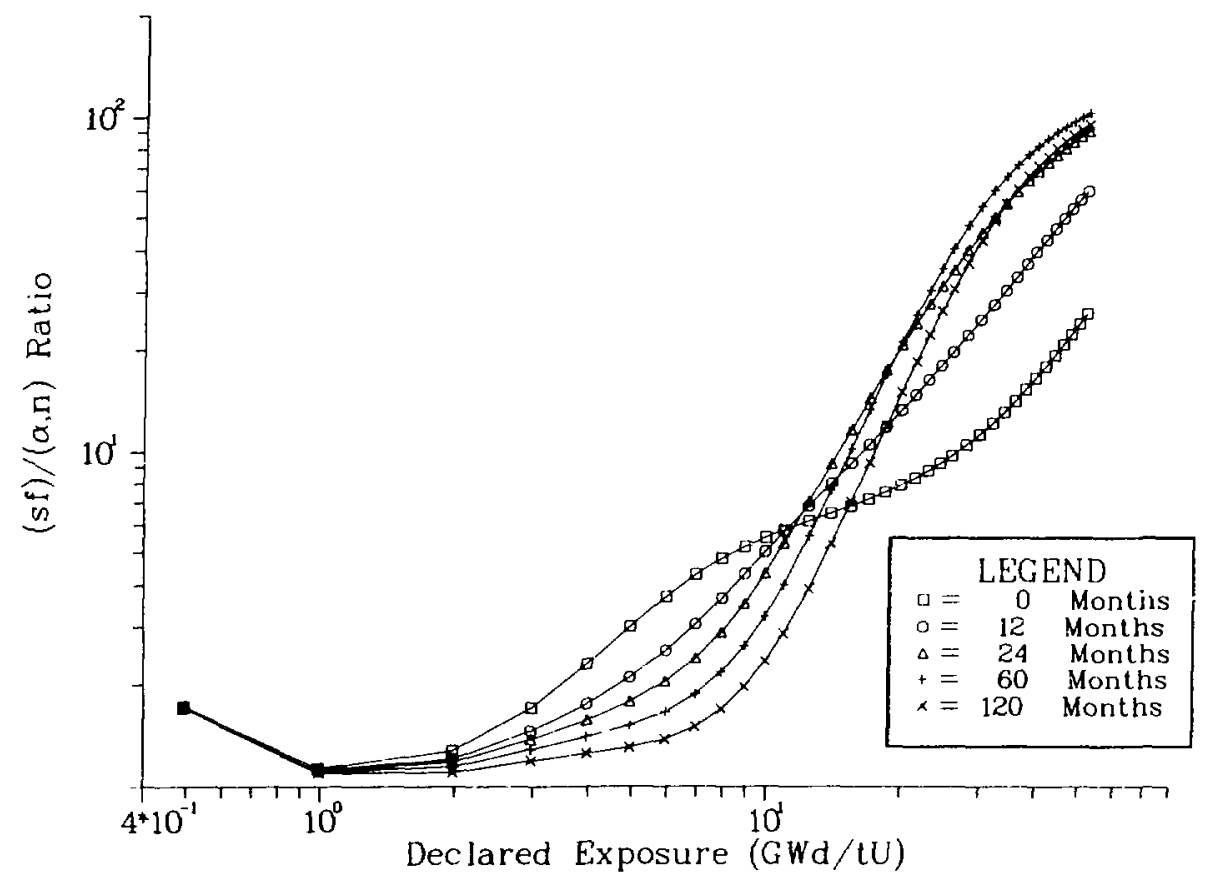

Fig. 7.

The ratio of spontaneous fission to $(\alpha, n)$ neutrons is shown at selected cooling times and over an exposure range (including some very low values). The change in the effect of cooling time mentioned in the caption of Fig. is is reflected here by the crossing of the curves just above $10 \mathrm{GWd} / \mathrm{tU}$. 

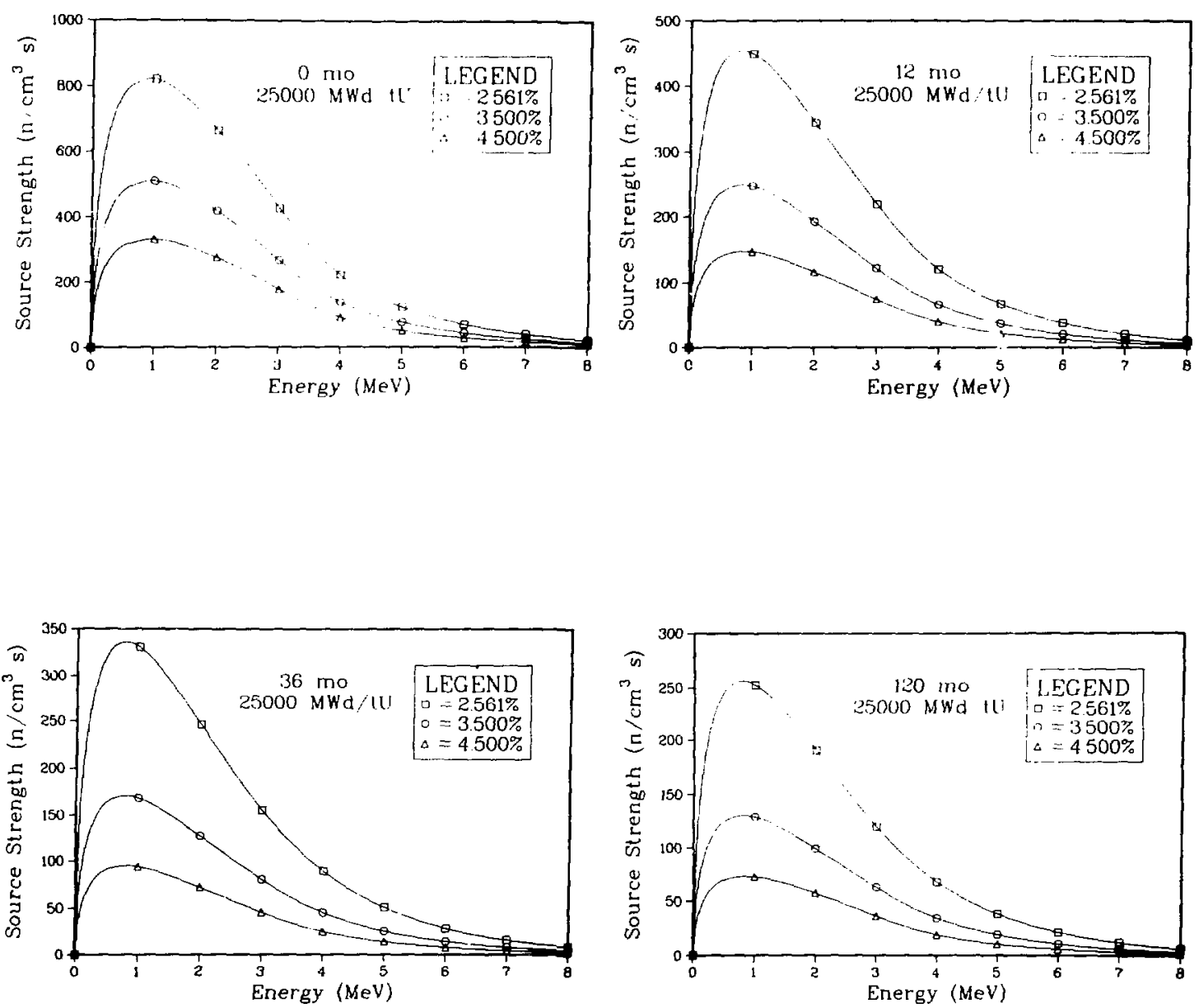

Fig. 8.

The source strength (but not the spectral shape) is strongly affected by the initial enrichment. Only one exposure is shown here, but the curves are typical of all exposures studied. It should be noted that the linear power density was a constant throughout this study, so when the enrichment was higher, the neutron flux was lower. This means that the $4.5 \%$ curves always correspond to weaker source strengths in this figure. 

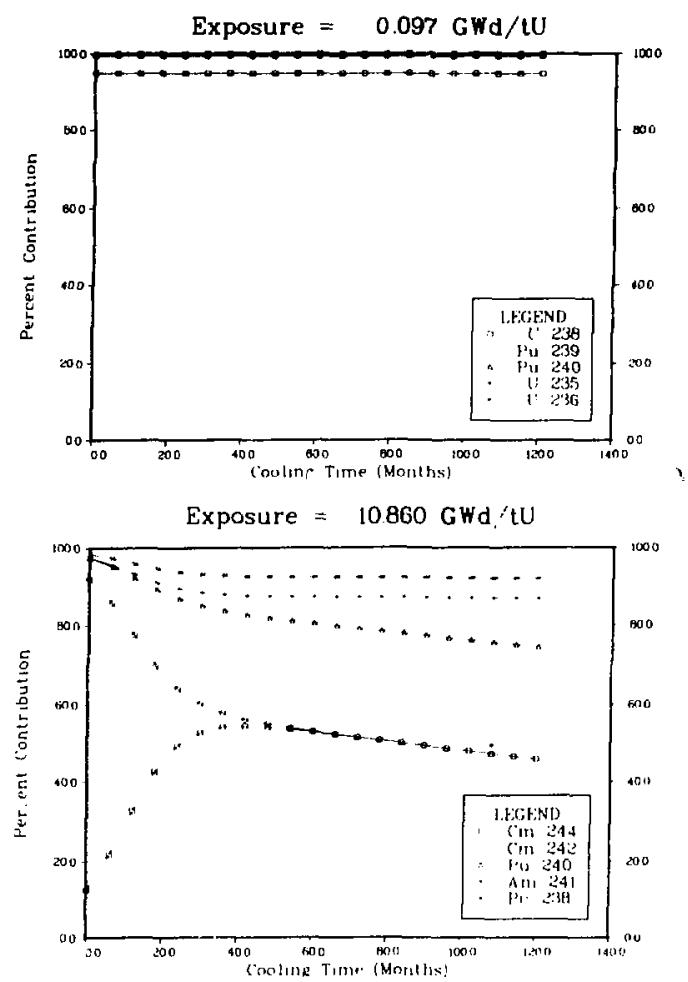

Exposure $=25.618 \mathrm{GWd} / \mathrm{tU}$

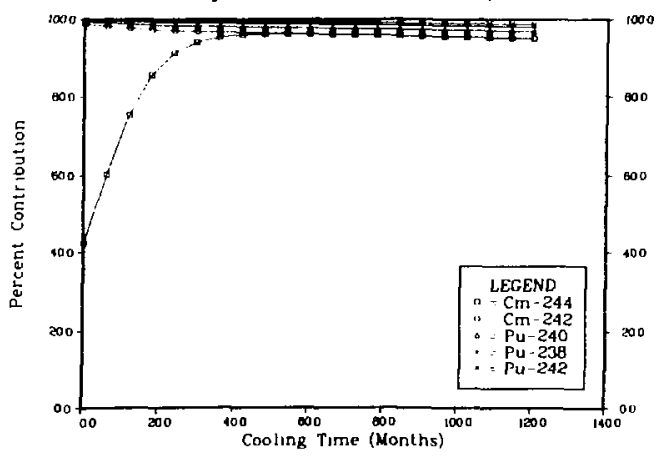

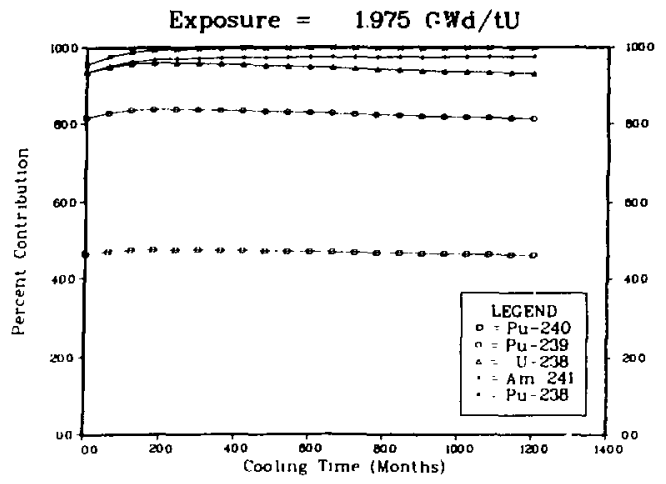
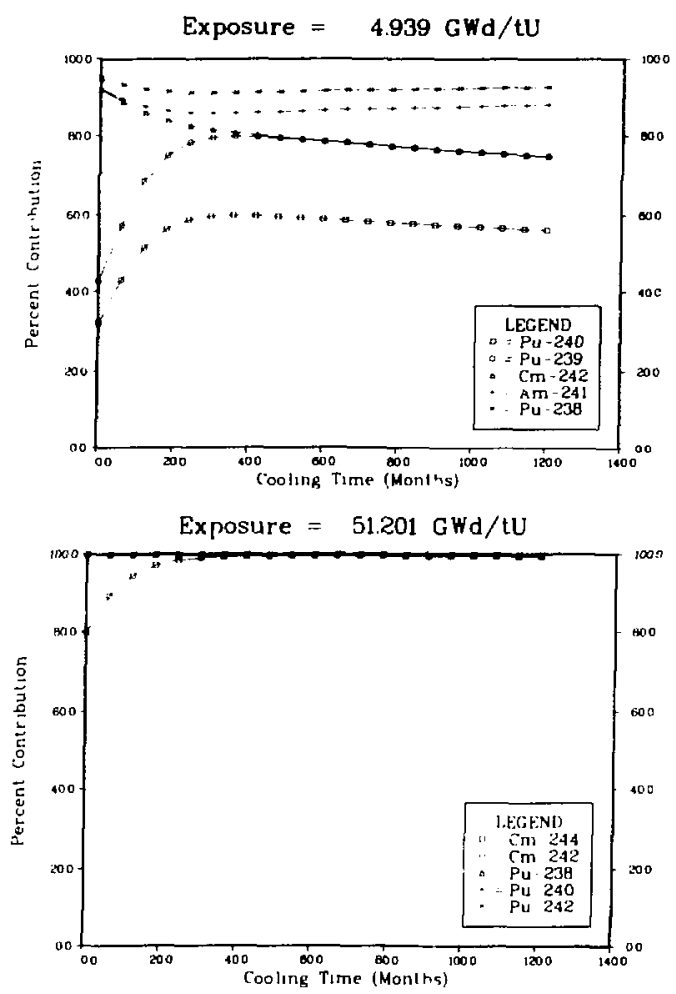

Fig. 9.

The five most important contributors to the number of neutrons emitted per cubic centimeter per second are shown as functions of cooling time after representative exposures. Along any vertical line of constant cooling time, the distance between two consecutive curves is the per cent contribution from the isotope identified with the upper curve. The dominant instigator of neutrons shifts from uranium, to plutonium, and then to curium isotopes as the exposure increases. 